\title{
Association of Menstrual Extension and Surgery Effectiveness with Ultrasound Parameters of Cesarean Section Scar Diverticulum in Patients Undergoing Transvaginal Uterine Diverticulum Repair
}

\author{
Qing Yang, Min Ren, Xiaoli Lv, and Fenghua Chen $(\mathbb{D}$ \\ Shanghai First Maternity and Infant Hospital, Tongji University School of Medicine, Shanghai, China \\ Correspondence should be addressed to Fenghua Chen; 13585852978@163.com
}

Received 9 July 2019; Accepted 26 November 2019; Published 19 December 2019

Academic Editor: Jacek Cezary Szepietowski

Copyright (c) 2019 Qing Yang et al. This is an open access article distributed under the Creative Commons Attribution License, which permits unrestricted use, distribution, and reproduction in any medium, provided the original work is properly cited.

\begin{abstract}
The association of residual myometrium thickness (RMT) and scar defect depth $(D)$ with menstrual abnormalities and the effectiveness of vaginal repair remain to be determined in patients with cesarean section scar diverticulum (CSD). To assess the value of ultrasound to predict vaginal repair effectiveness. This was a retrospective study of patients with CSD treated with vaginal repair between 01/2014 and 02/2016 at Shanghai First Maternity and Infant Hospital (Tongji University). Transvaginal ultrasound was performed before and 3 months after surgical repair. RMT, $D$, scar defect length $(L)$, and scar defect width $(W)$ were measured. Width $(W), D$, and $L$ increased along the duration of menstrual period $(P<0.05)$. When the menstrual extension time was $\geq 15$ days, $\mathrm{RMT} / D$ and $\mathrm{RMT} /(\mathrm{RMT}+D)$ were smaller than in patients with period $<15$ days $(P<0.05) . L$ was the most positively correlated ultrasonic parameter with menstrual prolongation $(r=0.492)$. RMT/D and RMT/(RMT $+D)$ were negatively correlated with prolonged menstruation $(r=-0.304$ and -0.305 , respectively). RMT/D and $\mathrm{RMT} /(\mathrm{RMT}+D)$ were associated with the disappearance of CSD after vaginal repair $(P<0.05)$. The cutoff value of RMT/ $R M T+D)$ was 0.496 , with sensitivity of $53.0 \%$ and specificity of $61.4 \%$. L of CSD is closely correlated with menstrual extension but has no relationship with the effectiveness of surgery. $\mathrm{RMT} /(\mathrm{RMT}+D)$ is correlated with menstrual extension time $\geq 15$ days and the effectiveness of vaginal repair.
\end{abstract}

\section{Introduction}

In 1985, the World Health Organization (WHO) proposed that the ideal cesarean section (CS) rate should be 10\%-15\%, but over the past 30 years, the CS rate has gradually increased worldwide [1]. Despite the fact that CS is often necessary to save the neonate and the mother, many CS are performed for nonmedical reasons [2]. This is of concern because CS can result in many complications such as uterine rupture during the following pregnancy, chronic pelvic pain, and CS scar diverticulum (CSD) $[3,4]$. CSD can cause prolonged menstrual period, menorrhagia, dysmenorrhea, and infertility $[5,6]$.

It is globally accepted that the first choice for assessing the CS scar is transvaginal ultrasonography $[7,8]$. Vaginal repair of CSD is a common surgical method to restore the uterine anatomical morphology. Wang et al. [6] observed that abnormal symptoms of CSD were related to the width $(W)$ of the scar defect but were unrelated with the residual myometrium thickness (RMT) and scar defect depth $(D)$, and later agreed that the equation RMT/(RMT $+D)$ offers additional information on the correlation between defect size and clinical symptoms [9].

Some studies have been reported the relationship between CSD size and menstrual abnormalities [5, 10-13], but beside the study by Wang et al. [6], little information is available on the relationship between menstrual abnormalities and RMT and $D$, as summarized by the equation $\mathrm{RMT} /(\mathrm{RMT}+D)$. The exact association of RMT/ $\mathrm{RMT}+D)$ with menstrual abnormalities and the effectiveness of vaginal repair remain to be determined.

Therefore, the purpose of the present study was to examine the relationship between ultrasound parameters of CSD and menstrual abnormalities and to assess whether the ultrasound parameters could be used to predict the effectiveness of vaginal repair. 


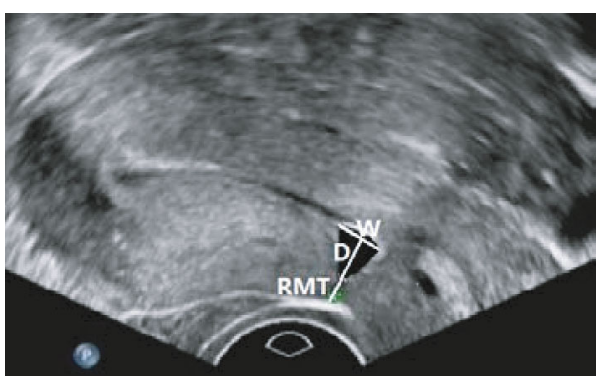

(a)

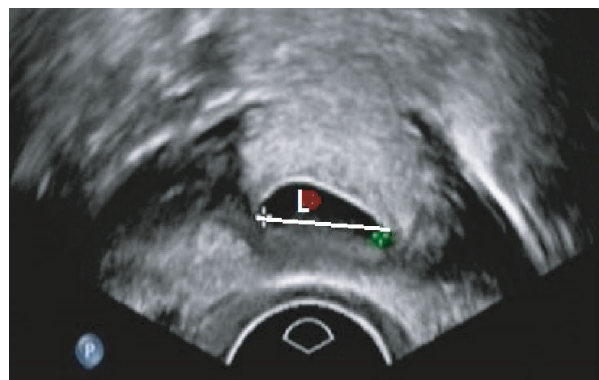

(c)

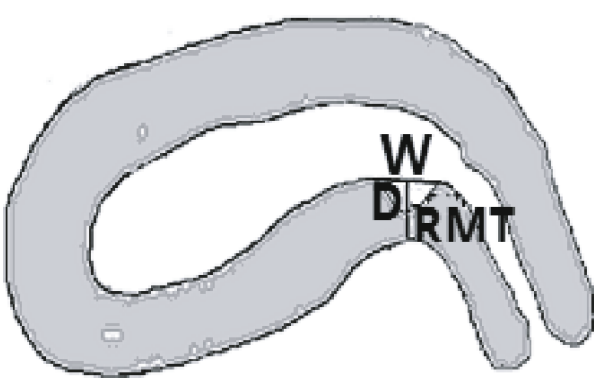

(b)

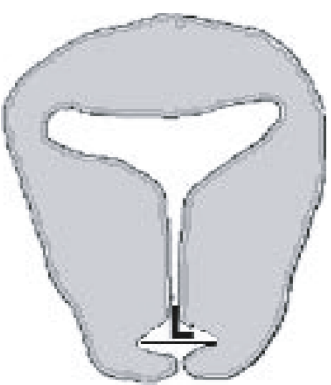

(d)

FIGURE 1: Ultrasonographic imaging of CSD before transvaginal repair surgery. (a) Ultrasound imaging of a cesarean scar diverticulum (CSD) on the sagittal plane. (b) Schematic diagram of the CSD on the sagittal plane. (c) Ultrasound imaging of a CSD on the transverse plane. (d) Schematic diagram of the CSD on the transverse plane. $W$ : width of the scar niche on the sagittal plane; $D$ : depth of the scar niche on the sagittal plane; RMT: residual myometrial thickness on the sagittal plane; $L$ : length of the scar niche on the transverse plane.

\section{Methods}

2.1. Study Design and Patients. This was a retrospective study of patients with CSD treated with vaginal repair between January 2014 and February 2016 at the Shanghai First Maternity and Infant Hospital affiliated to Tongji University. This study was approved by the Ethics Committee of the Shanghai First Maternity and Infant Hospital affiliated to Tongji University. The need for individual consent was waived by the committee.

The inclusion criteria were the following: (1) underwent at least one CS delivery, (2) CSD diagnosis by transvaginal ultrasound, and (3) underwent vaginal repair. The exclusion criteria were the following: (1) patients with any history of irregular periods, (2) use of intrauterine device, (3) use of hormonal contraceptives, (4) coagulation disorders, or (5) with any other uterine disease.

\subsection{Ultrasound Examinations. Ultrasound examinations} were performed using a Philips HD15 (US) system (Philips, Best, Netherlands) or a GE Voluson E8 system (GE Healthcare, Zipf, Austria). Both ultrasound devices were equipped with a $4-9 \mathrm{MHz}$ transvaginal probe. The patient was in the lithotomy position after emptying the bladder. The transvaginal probe was used to gently touch the cervix to measure the uterine size and endometrial thickness. The multisection dynamic scan was performed to observe the location and morphology of the uterus, myometrium, endometrial echo, morphological changes, whether there were abnormal masses and effusions in the uterine cavity, and the presence or absence of abnormal echo in parametrial tissues. The characteristics of the CS scar at the lower segment of the uterine anterior wall (including the presence or absence of CSD, morphology of CSD, internal echo, relation with uterine cavity, and continuity of the muscular and serosal layers) were recorded. Auxiliary color Doppler ultrasonography was also performed.

Transvaginal ultrasound was routinely performed twice: before surgery and 3 months after surgical repair. For standardization purposes, the terms proposed by Naji et al. [8] were used in the present study. All ultrasound reports and images were reviewed to ensure the consistency of the definitions and values. The standardized approaches for imaging and measuring CSD were used, as described by Pomorski et al. [14] and Naji et al. [8]. Three parameters were measured on the sagittal plane: width of the hypoechoic scar niche $(W)$, depth of the hypoechoic scar niche (D), and RMT (Figures 1(a) and 1(b)). The length of the hypoechoic scar niche $(L)$ was measured on the transverse plane (Figures $1(\mathrm{c})$ and $1(\mathrm{~d})$ ). In addition, $\mathrm{RMT} / D$ and $\mathrm{RMT} /(\mathrm{RMT}+D)$ were calculated.

2.3. Vaginal Repair. Transvaginal repair surgery was performed according to the previous studies $[11,15,16]$. All operations were performed by a chief professor with extensive experience in gynecological surgery. The patients underwent transvaginal diverticular repair and received transvaginal ultrasound examination 3 months after surgery to confirm the success of surgery. According to whether the anatomical structure of the cesarean section was restored, the patients were classified into two groups: the cured group (no diverticulum) and the unhealed group (with residual diverticulum). 
2.4. Statistical Analysis. Statistical analysis was performed using SPSS 22.0 for Windows (IBM, Armonk, NY, USA). Continuous data are presented as means \pm standard deviation or medians (range) and were analyzed using oneway analysis of variance (ANOVA) test or Kruskal-Wallis $H$ test (intragroup comparisons) and the Student $t$-test or Mann-Whitney $U$ test (intergroup comparisons), based on the results of the Kolmogorov-Smirnov test for normal distribution. Categorical data were presented as number and percentages and were analyzed using the chi-square test or the Fisher exact test, as appropriate. Correlations were analyzed with Spearman's rank correlation coefficient. The receiver operating characteristic (ROC) curve analysis was performed to determine the cutoff values. $P$ values $<0.05$ were considered statistically significant.

\section{Results}

3.1. Patient Characteristics. The study included 241 women with a mean age of $32.9 \pm 3.7$ (from 24 to 42 ) years. Among those, 177 (73.4\%) women had undergone one CS, 61 (25.3\%) had undergone two CS, and 3 (1.24) had undergone three CS. There were $230(95.4 \%)$ patients with prolonged menstrual period, 8 (3.3\%) with normal menstruation, 2 $(0.8 \%)$ with midmenstrual menorrhagia, 1 (0.4\%) with shortened menstrual cycle, and $3(1.24 \%)$ with other conditions ( 2 had midmenstrual hemorrhage, and one had shortened period) (Table 1).

3.2. Ultrasound. The shape of the diverticulum was roughly divided into five types: triangle $(n=102,42.3 \%)$, wedge shape $(n=67,27.8 \%)$, quasi-circular $(n=38,15.8 \%)$, droplet $(n=19,7.9 \%)$, and irregular shape $(n=15,6.2 \%)$. The uterine position was categorized as retroflexion $(n=141$, $58.5 \%)$, anteflexion $(n=91,37.8 \%)$, and neutral position $(n=9,3.7 \%)$ (Figure 2).

3.3. Factors Associated with Prolonged Menstruation. The patients with menstrual prolongation were divided into three subgroups: menstrual time 8-10 days, 11-14 days, and $\geq 15$ days. The associations between prolonged menstrual bleeding, age, number of CS, and ultrasound parameters of CSD are presented in Table 2. The number of CS was higher in women with menstruations $\geq 15$ days. $D, L$, and $W$ progressively increased with the length of the menstrual period. $\mathrm{RMT} / D$ and $\mathrm{RMT} /(\mathrm{RMT}+D)$ were smaller in patients with menstrual period $\geq 15$ days.

3.4. Correlation between Ultrasound Parameters of CSD and Prolonged Menstruation. $L$ was positively correlated with menstrual extension $(r=0.492)$. RMT/D and RMT/ $(\mathrm{RMT}+D)$ were negatively correlated with prolonged menstrual period $(r=-0.304)$ (Table 3$)$.

3.5. Ultrasound Parameters of CSD and Surgical Repair Outcomes. After surgical repair, 124 patients' CSD disappeared while 117 patients' CSD remained. Ultrasonographic imaging of CSD after transvaginal repair surgery is shown in Figure 3.
TABLE 1: Characteristics of the patients.

\begin{tabular}{|c|c|c|}
\hline Parameters & $n$ & Value \\
\hline Age (years) & 241 & $32.9 \pm 3.7$ \\
\hline \multicolumn{3}{|l|}{ Number of CS } \\
\hline 1 & 177 & \\
\hline 2 & 61 & \\
\hline 3 & 3 & \\
\hline \multicolumn{3}{|l|}{ Age of CS (years) } \\
\hline 1 & 241 & $26.4 \pm 3.8$ \\
\hline 2 & 61 & $28.7 \pm 3.7$ \\
\hline 3 & 3 & $27.5 \pm 2.1$ \\
\hline Menstrual before first CS (days) & 241 & $6.2 \pm 1.0$ \\
\hline \multicolumn{3}{|l|}{ Menstrual changes } \\
\hline Prolonged bleeding & 230 & $14.4 \pm 3.3$ \\
\hline Normal & 8 & \\
\hline Other & 3 & \\
\hline \multicolumn{3}{|l|}{ Uterine position } \\
\hline Retroflexion & 141 & \\
\hline Anteflexion & 91 & \\
\hline Neutral position & 9 & \\
\hline Last CS to surgery interval (years) & 241 & $5.0 \pm 2.8$ \\
\hline
\end{tabular}

CS: cesarean section.

Analysis of factors related to CSD existence or disappearance after surgery is performed in Table 4 . There were significant differences in $\mathrm{RMT} / D(P=0.048)$ and $\mathrm{RMT} /(\mathrm{RMT}+\mathrm{D})$ $(P=0.048)$ before surgery between the absence or presence of CSD after surgery, but no differences in the number of CS, uterine position, $W, D, L$, and RMT $(P<0.05)$.

ROC curve was used to calculate the cutoff values of $\mathrm{RMT} /(\mathrm{RMT}+\mathrm{D})$ for the absence or presence of CSD. $\mathrm{RMT} /(\mathrm{RMT}+\mathrm{D})>0.496$ indicated that the likelihood of CSD disappearance after VR was greater than 50\%, with sensitivity of $53.0 \%$ and specificity of $61.4 \%$.

\section{Discussion}

The association of RMT and D with menstrual abnormalities and the effectiveness of vaginal repair remain to be determined in patients with CSD. Therefore, the aim of the present study was to examine the relationship between ultrasound of CSD and menstrual abnormalities and to assess the value of ultrasound to predict vaginal repair effectiveness. The results suggest that the $L$ of CSD is correlated with menstrual extension but has no relationship with CSD disappearance or existence after vaginal repair. $\mathrm{RMT} /(\mathrm{RMT}+\mathrm{D})$ is correlated with menstrual extension time $\geq 15$ days and the effectiveness of vaginal repair.

CS is an important means of dealing with high-risk pregnancies and solving difficult births [1]. Nevertheless, there are potential long-term risks for subsequent pregnancy such as scar pregnancy, placenta previa, and uterine rupture $[3,4]$. In recent years, the incidence of CS has gradually increased [1]. A survey of 39 hospitals in 


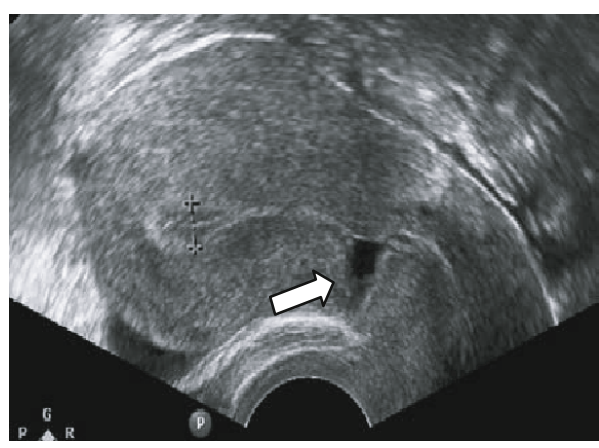

(a)

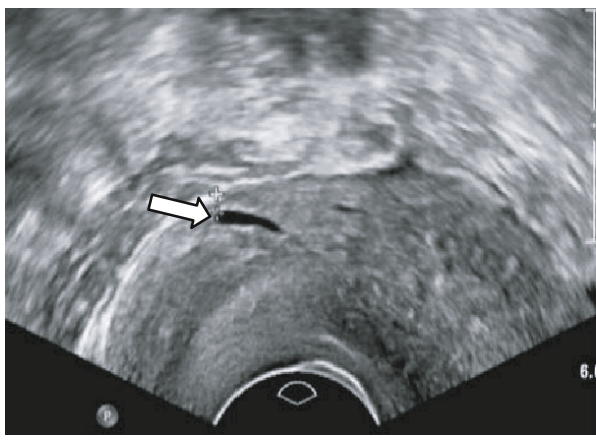

(c)

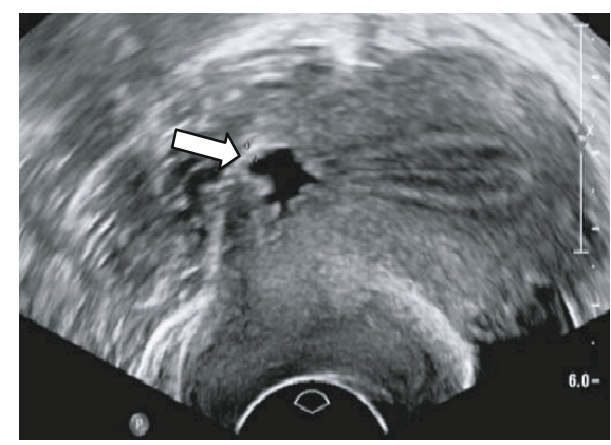

(b)

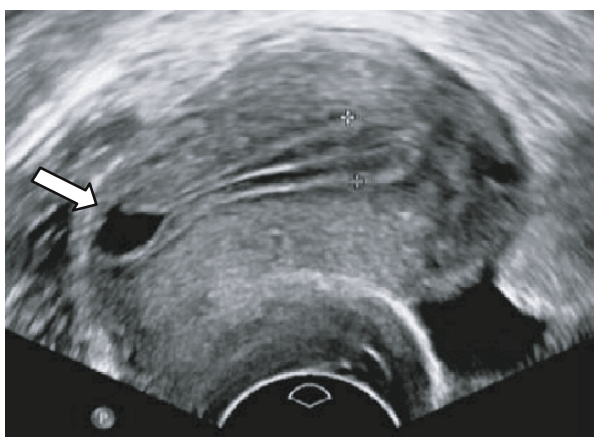

(d)

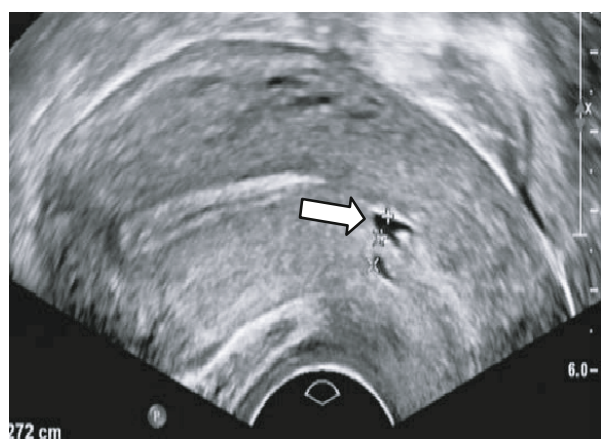

(e)

Figure 2: Shapes of the cesarean section diverticulum. (a) Triangle shape. (b) Wedge shape. (c) Quasicircular shape. (d) Droplet shape. (e) Irregular shape.

Mainland China showed that the incidence of CS is $54.5 \%$, among which the CS rate without indication was $24.6 \%$ [17]. With the increase of cesarean section rate, the incidence of CSD also increases gradually, which is reported to be as high as $62.5 \%$ among women with at least one CS [17].

The factors leading to poor CS scar healing are unclear. From nine studies, Melo-Cerda et al. classified all risk factors into four main categories: closure technique, development of the lower uterine segment or location of the incision, wound healing, and miscellaneous [18]. The risk of CS scar increases in women with a retroflexed uterus and in those who had undergone multiple CS [19]. Wang et al. [6] found that multiple CS and retroflexed uterus were risk factors for larger CSD. In the study by Osser et al. [20], the frequency of large scar defects increased with the number of CS [20]. Some authors suggested that CSD development was significantly associated with premature rupture of membrane and short operation time [21]. In the present study, 141 cases of retroflexed uterus $(58.5 \%)$ were reported.

Currently, there is no uniform diagnostic standard for CSD. For patients with clinical symptoms, it is necessary to exclude endocrine factors and examine the uterus carefully to make a diagnosis. At present, the examinations of CSD include transvaginal ultrasound, magnetic resonance imaging, hysteroscopy, hysterosalpingography, and saline contrast sonohysterography. Transvaginal ultrasound can be used to measure and describe the morphology of CS scars when present $[7,8]$. Transvaginal ultrasound is a first-line clinical tool for the diagnosis of abnormal uterine bleeding [22] and is the first choice for the noninvasive examination of CSD $[5,23]$. In the present study, the CS scars in transvaginal ultrasound were classified into triangle (42.3\%), wedge (27.8\%), quasicircular, droplet, and irregular shapes. Park et al. [21] reported that the triangle and quasicircular shapes were the most common, supporting the present study. 
TABLE 2: Relationship between prolonged menstrual bleeding, age, number of CS, and ultrasound parameters of CSD.

\begin{tabular}{|c|c|c|c|c|}
\hline & $\begin{array}{c}8-10 \text { days } \\
N=56\end{array}$ & $\begin{array}{c}11-14 \text { days } \\
N=78\end{array}$ & $\begin{array}{c}\geq 15 \text { days } \\
N=96\end{array}$ & $P$ \\
\hline Age (years) (mean $\pm S D)$ & $32.3 \pm 3.4$ & $33.1 \pm 3.9$ & $33.2 \pm 3.9$ & 0.303 \\
\hline Number of CS & & & & 0.008 \\
\hline 1 & 43 & 65 & 59 & \\
\hline 2 & 13 & 13 & 34 & \\
\hline 3 & 0 & 0 & 3 & \\
\hline Uterine position & & & & 0.108 \\
\hline Retroflexion & 35 & 50 & 47 & \\
\hline Anteflexion & 21 & 24 & 45 & \\
\hline Neutral position & 0 & 4 & 4 & \\
\hline$W$ & $5(4-7)$ & $7(6-9)^{\#}$ & $8(6-11)^{\#, *}$ & $<0.001$ \\
\hline$D$ & $5(4-7)$ & $7(5-8)^{\#}$ & $8.5(6-10)^{\#, *}$ & $<0.001$ \\
\hline$L$ & $9.3 \pm 3.9$ & $12.5 \pm 4.1^{\#}$ & $16.6 \pm 4.7^{\#, *}$ & $<0.001$ \\
\hline $\mathrm{RMT}$ & $2.4(2-3.42)$ & $2.4(2-3)$ & $2.3(1.7-2.92)$ & 0.177 \\
\hline $\mathrm{RMT} / D$ & $0.50(0.33-0.75)$ & $0.40(0.27-0.57)$ & $0.28(0.19-0.43)^{\# *}$ & $<0.001$ \\
\hline $\mathrm{RMT} /(\mathrm{RMT}+D)$ & $0.33(0.25-0.43)$ & $0.29(0.21-0.36)$ & $0.22(0.16-0.30)^{\#, *}$ & $<0.001$ \\
\hline
\end{tabular}

CS: cesarean section; CSD: cesarean section scar diverticulum; $W$ : width of the niche; $D$ : depth of the niche; $L$ : length of the niche; RMT: residual myometrial thickness. ${ }^{\#} P<0.05$ vs. 8 - 10 days and ${ }^{*} P<0.05$ vs. $11-14$ days.

TABLE 3: Correlation coefficients of variables related to prolonged menstruation.

\begin{tabular}{lcc}
\hline$N=230$ & Correlation coefficient & $P$ \\
\hline Number of CS & 0.193 & 0.003 \\
$W$ & 0.323 & $<0.001$ \\
$D$ & 0.327 & $<0.001$ \\
$L$ & 0.492 & $<0.001$ \\
$\mathrm{RMT} / D$ & -0.305 & $<0.001$ \\
$\mathrm{RMT} /(\mathrm{RMT}+D)$ & -0.304 & $<0.001$ \\
\hline
\end{tabular}

CS: cesarean section; $W$ : width of the niche; $D$ : depth of the niche; $L$ : length of the niche; RMT: residual myometrial thickness.

Using vaginal ultrasonography, Wang et al. [6] observed that the $W$ of CSD was associated with postmenstrual spotting, but was unrelated to the RMT, supporting the present study. We also found that the $W, D$, and $L$ of CSD were related to menstrual extension. In other words, the wider, the deeper, and the longer the CSD is, the longer the length of menstrual prolongation is. Among those, $L$ was the most closely related to the duration of menstrual prolongation. Interestingly, we also found that although RMT had no relationship with prolonged menstruation, $\mathrm{RMT} / D$ and $\mathrm{RMT} /(\mathrm{RMT}+D)$ were associated with prolonged menstruation $\geq 15$ days.

The methods for the surgical treatment of CSD include transvaginal diverticulum repair, hysteroscopy, and laparoscopy. Transvaginal repair is an effective minimally invasive surgical treatment $[10,15,24-26]$. Among the studies about the factors associated with surgical success, only one suggested that multiple CS and CSD volume $>600 \mathrm{~mm}^{3}$ measured by magnetic resonance imaging were risk factors for surgical failure [27]. Pomorski et al. [14] reported that $D / \mathrm{RMT}$ was useful to predict the occurrence of scar dehiscence in the next pregnancy. In the present study, $\mathrm{RMT} / D$ could also predict the surgical outcome: the larger RMT and the smaller $D$ are, the greater the possibility of surgical cure is. We were surprised to find that none of the assessed CSD parameters individually was useful for predicting outcome of surgery. One possible explanation is that RMT and $D$ values together represent the complete muscular thickness of the scar site of a CS. $\mathrm{RMT} /(\mathrm{RMT}+\mathrm{D})$ can be calculated to provide an indication of the percentage of RMT at the exact location of the CS scar.

This study has limitations. The sample size was relatively small and from a single center, and all were operated by the same surgeon. The clinical experience and suturing techniques of surgeons during transvaginal repair are central to surgical success [28], and they cannot be measured using standard quantitative indicators. Furthermore, the vascular perfusion oxygenation in the scar tissue [29] may need to be taken into account in future studies on the effectiveness of CSD surgery.

\section{Conclusions}

The results of the present study suggest that $\mathrm{RMT} /(\mathrm{RMT}+\mathrm{D})$ is an important parameter in ultrasound assessment of $\mathrm{CSD}$. $\mathrm{RMT} /(\mathrm{RMT}+D)$ is related not only to prolonged menstruation $\geq 15$ days but also to the outcome of transvaginal CSD repair. Those findings may help in counselling for clinical symptoms and surgical decision of patients with CSD. Future studies on surgical may need to take these into account. 


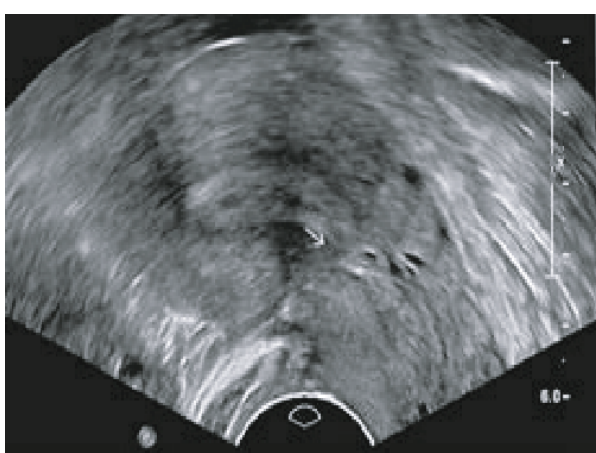

(a)

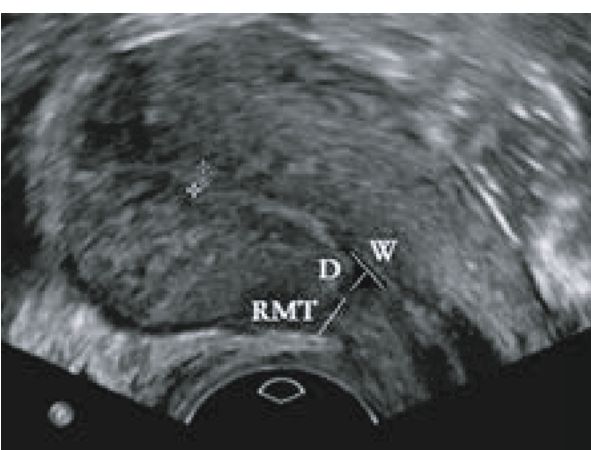

(b)

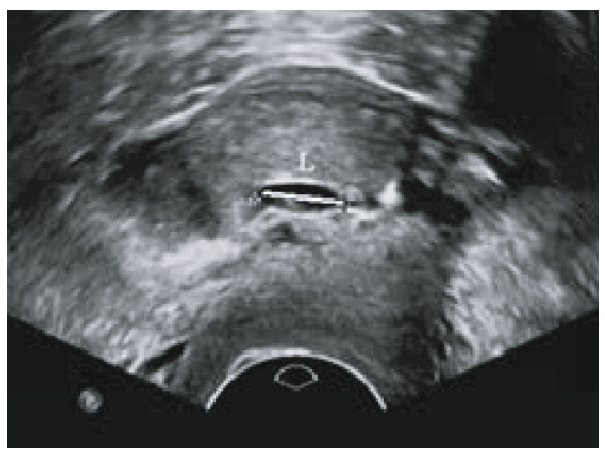

(c)

FIGURE 3: Ultrasonographic imaging of cesarean scar diverticulum (CSD) after transvaginal repair surgery. (a) Ultrasound imaging of CSD disappearance on the sagittal plane. No defect is seen in the scar shown by the arrow. (b) Ultrasound imaging showing CSD remained on the sagittal plane. (c) Ultrasound imaging showing CSD remained on the transverse plane. W: width of the scar niche on the sagittal plane; $D$ : depth of the scar niche on the sagittal plane; RMT: residual myometrial thickness on the sagittal plane; $L$ : length of the scar niche on the transverse plane.

TABLE 4: Characteristics of CSD disappearance and existence after vaginal repair.

\begin{tabular}{|c|c|c|c|}
\hline Variables & $\begin{array}{l}\text { CSD disappearance after VR } \\
\qquad N=124\end{array}$ & $\begin{array}{l}\text { CSD existence after VR } \\
\qquad N=117\end{array}$ & $P$ \\
\hline Age (years) $($ mean $\pm S D)$ & $32.8 \pm 3.8$ & $33.1 \pm 3.7$ & 0.495 \\
\hline Number of CS & & & 0.909 \\
\hline 1 & 92 & 86 & \\
\hline 2 & 31 & 29 & \\
\hline 3 & 1 & 2 & \\
\hline Uterine position & & & 0.29 \\
\hline Retroflexion & 73 & 68 & \\
\hline Anteflexion & 49 & 42 & \\
\hline Neutral position & 2 & 7 & \\
\hline$W$ & $7(5-9)$ & $7(5-11)$ & 0.147 \\
\hline$D$ & $7(5-9)$ & $7(5.5-9)$ & 0.237 \\
\hline$L$ & $12.9 \pm 5.2$ & $13.8 \pm 5.3$ & 0.196 \\
\hline RMT & $2.5(2.0-3.0)$ & $2.2(1.8-3.0)$ & 0.088 \\
\hline $\mathrm{RMT} / D$ & $0.40(0.26-0.60)$ & $0.31(0.23-0.50)$ & 0.048 \\
\hline $\mathrm{RMT} /(\mathrm{RMT}+D)$ & $0.29(0.20-0.38)$ & $0.24(0.19-0.33)$ & 0.048 \\
\hline
\end{tabular}

CS: cesarean section; CSD: cesarean section scar diverticulum; W: width of the niche; D: depth of the niche; L: length of the niche; RMT: residual myometrial thickness. 


\section{Data Availability}

No data were used to support this study.

\section{Conflicts of Interest}

The authors declare that there is no conflict of interest regarding the publication of this paper.

\section{Acknowledgments}

The authors acknowledge the help of Zhou Yuan for the advice on experimental design. This study was funded by the Shanghai Municipal Medical and Health Discipline Construction Projects (No. 2017ZZ02015).

\section{References}

[1] A. A. Boatin, A. Schlotheuber, A. P. Betran et al., "Within country inequalities in caesarean section rates: observational study of 72 low and middle income countries," BMJ, vol. 360, p. k55, 2018.

[2] T. Lavender, G. J. Hofmeyr, J. P. Neilson, C. Kingdon, G. M. L. Gyte, and Cochrane Pregnancy and Childbirth Group, "Caesarean section for non-medical reasons at term," Cochrane Database of Systematic Reviews, vol. 3, 2012.

[3] S. Sobhy, D. Arroyo-Manzano, N. Murugesu et al., "Maternal and perinatal mortality and complications associated with caesarean section in low-income and middle-income countries: a systematic review and meta-analysis," Lancet, vol. 393, no. 10184, pp. 1973-1982, 2019.

[4] S. L. Sholapurkar, "Long-term complications of caesarean section - an inevitable consequence?," BJOG, vol. 121, no. 11, pp. 1445-1446, 2014.

[5] A. M. Tower and G. N. Frishman, "Cesarean scar defects: an underrecognized cause of abnormal uterine bleeding and other gynecologic complications," Journal of Minimally Invasive Gynecology, vol. 20, no. 5, pp. 562-572, 2013.

[6] C. B. Wang, W. W. C. Chiu, C. Y. Lee, Y. L. Sun, Y. H. Lin, and C. J. Tseng, "Cesarean scar defect: correlation between cesarean section number, defect size, clinical symptoms and uterine position," Ultrasound in Obstetrics \& Gynecology, vol. 34, no. 1, pp. 85-89, 2009.

[7] J. Fonda, "Ultrasound diagnosis of caesarean scar defects," Australasian Journal of Ultrasound in Medicine, vol. 14, no. 3, pp. 22-30, 2011.

[8] O. Naji, Y. Abdallah, A. J. bij de Vaate et al., "Standardized approach for imaging and measuring cesarean section scars using ultrasonography," Ultrasound in Obstetrics \& Gynecology, vol. 39, no. 3, pp. 252-259, 2012.

[9] H. F. Yazicioglu, "Assessment of cesarean section scars with transvaginal sonography," Ultrasound in Obstetrics and Gynecology, vol. 35, no. 2, pp. 250-251, 2010.

[10] H. Chen, H. Wang, J. Zhou, Y. Xiong, and X. Wang, "Vaginal repair of cesarean section scar diverticula diagnosed in nonpregnant women," Journal of Minimally Invasive Gynecology, vol. 26, no. 3, pp. 526-534, 2019.

[11] Y. Chen, Y. Chang, and S. Yao, "Transvaginal management of cesarean scar section diverticulum: a novel surgical treatment," Medical Science Monitor, vol. 20, pp. 1395-1399, 2014.
[12] T. Uppal, V. Lanzarone, and M. Mongelli, "Sonographically detected caesarean section scar defects and menstrual irregularity," Journal of Obstetrics and Gynaecology, vol. 31, no. 5, pp. 413-416, 2011.

[13] L. M. Borges, A. Scapinelli, D. D. de Baptista, U. G. Lippi, and R. G. Coelho Lopes, "Findings in patients with postmenstrual spotting with prior cesarean section," Journal of Minimally Invasive Gynecology, vol. 17, no. 3, pp. 361-364, 2010.

[14] M. Pomorski, T. Fuchs, and M. Zimmer, "Prediction of uterine dehiscence using ultrasonographic parameters of cesarean section scar in the nonpregnant uterus: a prospective observational study," BMC Pregnancy Childbirth, vol. 14, no. 1, p. 365, 2014.

[15] L. Luo, G. Niu, Q. Wang, H. Z. Xie, and S. Z. Yao, "Vaginal repair of cesarean section scar diverticula," Journal of Minimally Invasive Gynecology, vol. 19, no. 4, pp. 454-458, 2012.

[16] B. Jeremy, C. Bonneau, E. Guillo et al., "Uterine ishtmique transmural hernia: results of its repair on symptoms and fertility," Gynécologie, Obstétrique \& Fertilité, vol. 41, no. 10, pp. 588-596, 2013.

[17] L. Hou, G. Li, L. Zou et al., "Cesarean delivery rate and indications in mainland China: a cross sectional study in 2011," Zhonghua Fu Chan Ke Za Zhi, vol. 49, no. 10, pp. 728-735, 2014.

[18] I. Melo-Cerda, “Cesarean scar defect," Ginecología y Obstetricia de México, vol. 82, no. 8, pp. 530-534, 2014.

[19] D. Ofili-Yebovi, J. Ben-Nagi, E. Sawyer et al., "Deficient lowersegment cesarean section scars: prevalence and risk factors," Ultrasound in Obstetrics \& Gynecology, vol. 31, no. 1, pp. 7277, 2008.

[20] O. V. Osser, L. Jokubkiene, and L. Valentin, "High prevalence of defects in cesarean section scars at transvaginal ultrasound examination," Ultrasound in Obstetrics \& Gynecology, vol. 34, no. 1, pp. 90-97, 2009.

[21] I. Y. Park, M. R. Kim, H. N. Lee, Y. Gen, and M. J. Kim, "Risk factors for Korean women to develop an isthmocele after a cesarean section," BMC Pregnancy and Childbirth, vol. 18, no. 1, p. 162, 2018.

[22] O. V. Osser, L. Jokubkiene, and L. Valentin, "Cesarean section scar defects: agreement between transvaginal sonographic findings with and without saline contrast enhancement," Ultrasound in Obstetrics \& Gynecology, vol. 35, no. 1, pp. 7583, 2010.

[23] K. C. Wheeler and S. R. Goldstein, "Transvaginal ultrasound for the diagnosis of abnormal uterine bleeding," Clinical Obstetrics and Gynecology, vol. 60, no. 1, pp. 11-17, 2017.

[24] J. Zhou, M. Yao, H. Wang, W. Tan, P. Chen, and X. Wang, "Vaginal repair of cesarean section scar diverticula that resulted in improved postoperative menstruation," Journal of Minimally Invasive Gynecology, vol. 23, no. 6, pp. 969-978, 2016.

[25] Y. Zhang, "A comparative study of transvaginal repair and laparoscopic repair in the management of patients with previous cesarean scar defect," Journal of Minimally Invasive Gynecology, vol. 23, no. 4, pp. 535-541, 2016.

[26] M. Yao, W. Wang, J. Zhou et al., "Cesarean section scar diverticulum evaluation by saline contrast-enhanced magnetic resonance imaging: the relationship between variable parameters and longer menstrual bleeding," The Journal of Obstetrics and Gynaecology Research, vol. 43, no. 4, pp. 696-704, 2017. 
[27] H. Y. Xu, M. Y. Yang, X. Zhang et al., "Efficacy of caesarean scar defect repair in improving postmenstrual bleeding and factors associated with poor effect," Journal of Obstetrics and Gynaecology, vol. 37, no. 8, pp. 1076-1081, 2017.

[28] D. D. Lofrumento, M. A. di Nardo, M. de Falco, and A. di Lieto, "Uterine wound healing: a complex process mediated by proteins and peptides," Current Protein \& Peptide Science, vol. 18, no. 2, pp. 125-128, 2017.

[29] A. Setubal, J. Alves, F. Osório et al., "Treatment for uterine isthmocele, a pouchlike defect at the site of a cesarean section scar," Journal of Minimally Invasive Gynecology, vol. 25, no. 1, pp. 38-46, 2018. 


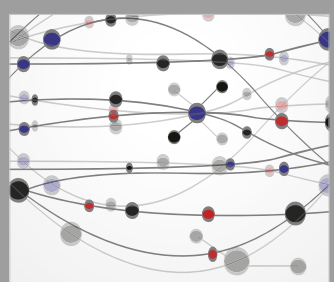

The Scientific World Journal
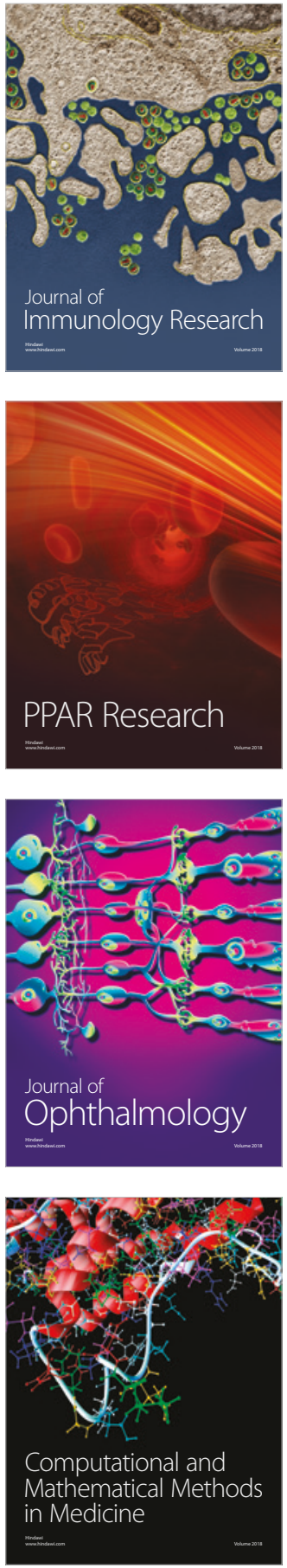

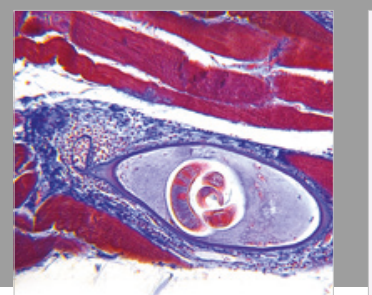

Gastroenterology Research and Practice

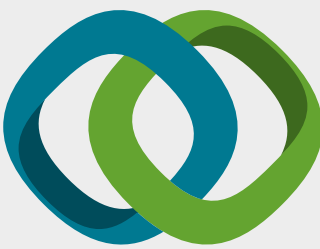

\section{Hindawi}

Submit your manuscripts at

www.hindawi.com
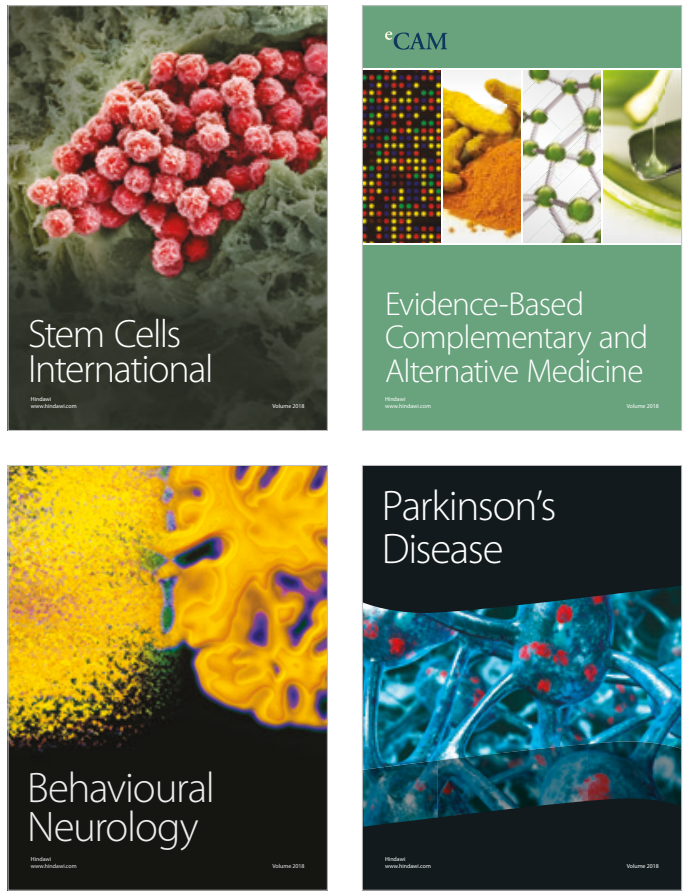

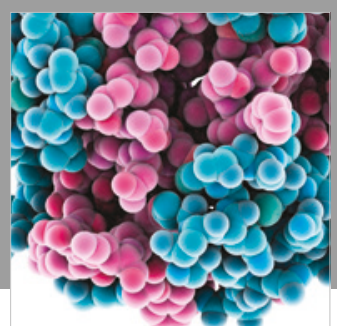

ournal of

Diabetes Research

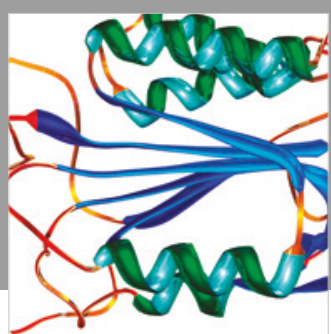

Disease Markers
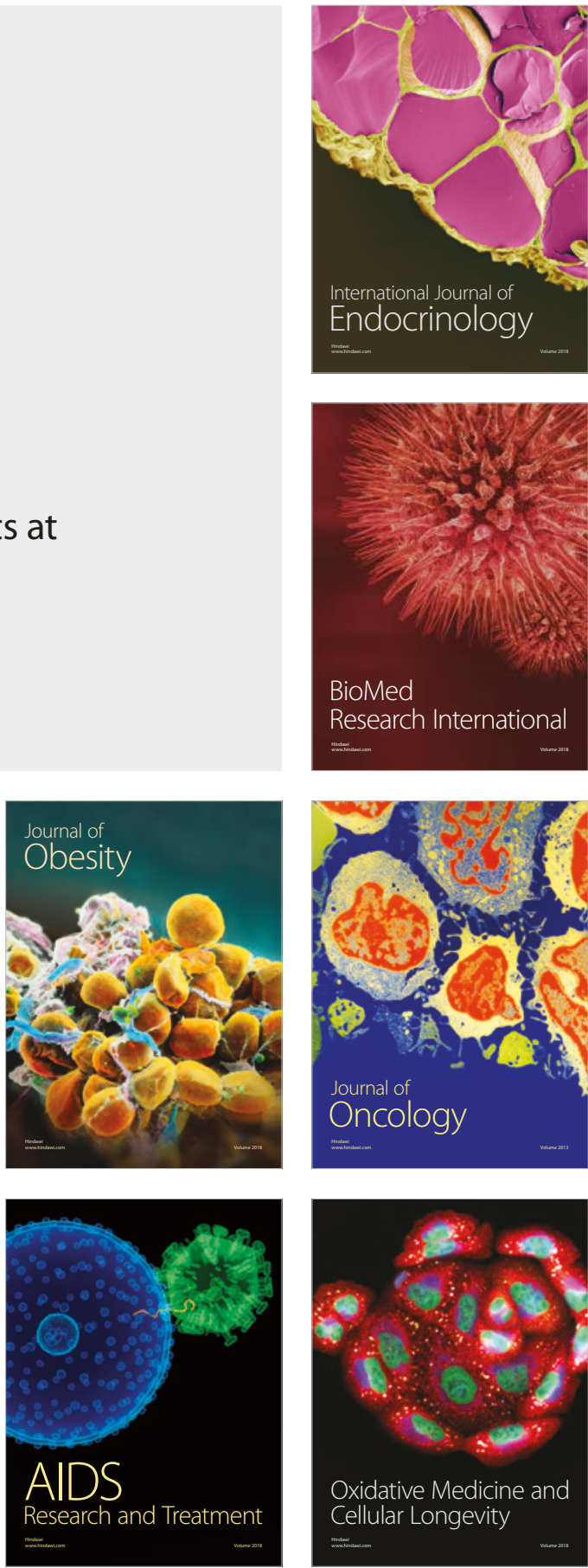